\title{
Identification and analysis of the FAD gene family in walnuts (Juglans regia L.) based on transcriptome data
}

\author{
Kai Liu ${ }^{1 \dagger}$, Shugang Zhao ${ }^{2^{*}+} \mathbb{B}$, Shuang Wang ${ }^{1}$, Hongxia Wang ${ }^{1,3,4^{*}}$ and Zhihua Zhang ${ }^{1,3,4^{*}}$
}

\begin{abstract}
Background: Walnut kernels contain a large amount of unsaturated fatty acids, such as linoleic acid and linolenic acid, which are essential fatty acids for humans and have important effects on growth and health. The main function of fatty acid desaturase (FAD), which is widely distributed in organisms, is to remove hydrogen from carbon chains in the biosynthesis of unsaturated fatty acids to generate $C=C$ bonds.

Results: By performing a series of bioinformatics analysis, 24 members of the JrFAD gene family were identified from the genome database of walnut, and then compared with the homologous genes from Arabidopsis. Phylogenetic analysis showed that JrFADs were classified into four subfamilies: the SAD desaturase subfamily, $\Delta 7 / \Delta 9$ desaturase subfamily, $\Delta 12 / \omega-3$ desaturase subfamily and "front-end" desaturase subfamily. Meanwhile, the expression of fatty acid synthesis genes in walnut kernels at different developmental stages was analysed by transcriptome sequencing, with expression of JrFAD3-1, which encodes an enzyme involved in linolenic acid synthesis, being particularly prominent. The relative expression level of JrFAD3-1 changed dramatically with the kernel development stages and exhibited a Bell-Shaped Curve. A significant positive correlation was observed between the expression of JrFAD3-1 during 70-100 DAF (Days after flowering) and the content of alpha-linolenic acid during 100-130 DAF, with a correlation coefficient of 0.991. Additionally, JrFAD3-1 was proved closely related to homologous genes in Betula pendula and Corylus heterophylla, indicating that the conserved structure of FADs is consistent with classical plant taxonomy.

Conclusion: Twenty-four members JrFADs in walnut were identified and classified into four subfamilies. JrFAD3-1 may play significant roles in the biosynthesis of polyunsaturated fatty acids in walnut.
\end{abstract}

Keywords: Walnut, Fatty acid, Gene expression, FAD gene family, FAD3

\section{Background}

Walnut (Juglans regia L.) is an important economic tree species throughout the world and is widely cultivated as a traditional wood and oil crop. The walnut kernel oil content can be as high as $63 \%$, higher than that of other

\footnotetext{
* Correspondence: zshug@hebau.edu.cn; whx@hebau.edu.cn; zzh@hebau.edu.cn

${ }^{\dagger}$ Kai Liu and Shugang Zhao contributed equally to this work.

${ }^{2}$ College of Life Sciences, Hebei Agricultural University, Baoding 071001, China

${ }^{1}$ Mountainous Area Research Institute of Hebei Province, Hebei Agricultural University, Baoding 071001, China

Full list of author information is available at the end of the article
}

leading oil crops, such as peanut, soybean, palm, olive, corn (as the germ), and sunflower [1]. Fatty acids, which are the main nutritional component accumulated in walnut kernels, comprise a large amount of unsaturated fatty acids, such as linoleic acid and linolenic acid [2], which are essential fatty acids for humans and have an important role in growth and health [3]. In addition, polyunsaturated fatty acids are prodrugs of prostaglandins, which have blood pressure-lowering, platelet adhesion-reducing, and anticoagulant effects [4]. However, the human body cannot synthesize linoleic acid and $\alpha$-linolenic acid, which can only be obtained

(c) The Author(s). 2020 Open Access This article is licensed under a Creative Commons Attribution 4.0 International License, which permits use, sharing, adaptation, distribution and reproduction in any medium or format, as long as you give appropriate credit to the original author(s) and the source, provide a link to the Creative Commons licence, and indicate if changes were made. The images or other third party material in this article are included in the article's Creative Commons licence, unless indicated otherwise in a credit line to the material. If material is not included in the article's Creative Commons licence and your intended use is not permitted by statutory regulation or exceeds the permitted use, you will need to obtain permission directly from the copyright holder. To view a copy of this licence, visit http://creativecommons.org/licenses/by/4.0/ The Creative Commons Public Domain Dedication waiver (http://creativecommons.org/publicdomain/zero/1.0/) applies to the data made available in this article, unless otherwise stated in a credit line to the data. 
through the diet [5]. The unsaturated fatty acid content of walnut kernel oil is as high as $90 \%$ [3], which can inhibit the absorption of cholesterol in the small intestine and the inhibit the re-absorption of bile acid, promote the degradation and elimination of cholesterol in the liver, alter the distribution of cholesterol in the body, and accelerate the transfer of plasma cholesterol to vascular tissues [4].

Fatty acid desaturase (FAD) is widely present in organisms [6-9]. The main function of FAD is to remove hydrogen from carbon chains in the biosynthesis of unsaturated fatty acids to produce $\mathrm{C}=\mathrm{C}$ bonds. In plants, FADs can be classified into two categories according to their solubility: soluble desaturases and membrane-integrins [10]. The stearoyl-ACP-desaturase (SAD) unique to higher plants is the only known FAD present in the matrix of plastids. All other types of desaturases belong to the integrin class and are localized to endoplasmic reticulum (FAD2 and FAD3) and plastid (e.g., FAD6, FAD7 and FAD8) membranes. The first step in the synthesis of unsaturated fatty acids is the introduction of a double bond at the $\Delta 9$ position [11], and the enzymes that catalyse the reaction include stearoyl-CoA-desaturase (SCD) [12] and SAD [13]. SAD, with the help of ferredoxin, uses the stearoyl carrier protein (stearoyl-ACP) to remove two hydrogens to form oleoyl ACP [14]. The second unsaturated bond is added by $\Delta 12$ desaturases (FAD2 and FAD6) based on the introduction of the first unsaturated bond [15]. $\triangle 15$ desaturases, such as FAD3, FAD7 and FAD8, play a key role in further decreasing the degree of saturation [16-18]. In particular, microsomal $\omega-3$ fatty acid desaturase, one of the FAD3 catalysed the synthesis of linolenic acid, is located in the endoplasmic reticulum and uses phospholipids as acyl substrates, and NADH, $\mathrm{NADH}$-cytochrome $b_{5}$ reductase and Cyt $b_{5}$ as electron donors [19].

The enzyme encoded by the FAD3 gene is a member of the $\omega-3$ fatty acid dehydrogenase family, a membrane integrin distributed in the endoplasmic reticulum that catalyses the introduction of a third unsaturated bond. The FAD3 amino acid sequence is highly conserved with three histidine-rich conserved domains, which together with divalent iron ions constitute the reaction centre of fatty acid dehydrogenase [20]. The Lys-Lys-X-X (KKXX) recovery signal contained at the $\mathrm{C}$-terminus of the amino acid sequence is consistent with the properties of endoplasmic reticulum integrins [20]. Since the first FAD3 was isolated from Arabidopsis [21], researchers have successively cloned genes from plants such as rapeseed, perilla, peony and Eucommia ulmoides. In recent years, the functional regulation of FAD3 in the synthesis and metabolism of unsaturated fatty acids has become a hot research topic.
With the release of walnut genome data, genes involved in the metabolism of walnut nutrients have been discovered. Indeed, a number of key genes encoding enzymes involved in walnut fatty acid metabolism, such as ACCase [22-24], have been cloned and characterized. In this research, walnut kernels at different stages of maturity were used as test materials to analyse the key gene family involved in unsaturated fatty acid synthesis based on bioinformatics analysis and transcriptome sequencing. This study explored the mechanism of walnut fatty acid formation, especially the anabolic mechanism of unsaturated fatty acids, and the results provide a theoretical basis for the regulation of walnut fatty acid synthesis. Furthermore, this study offers a theoretical reference for the rational development of walnut oil and oil-producing walnut cultivars.

\section{Results \\ Identification and analysis of FAD genes in the walnut genome}

We identified the FAD family using Arabidopsis FAD family protein sequence information to construct the hidden Markov model. The walnut protein data were searched, and 33 FAD family genes were screened. Using Pfam domain analysis, 24 FAD family genes were ultimately obtained, encoding 30 protein sequences, which are numbered according to their annotation in walnut (Table 1).

Because of the limited depth of analysis of walnut genome data, the specific distribution of these genes on the 16 pairs of chromosomes in walnut is unclear. The shortest length among these $F A D$ genes is $1262 \mathrm{bp}$, and the longest length is $14,280 \mathrm{bp}$. The CDS lengths of these genes ranged from $924 \mathrm{bp}-1368 \mathrm{bp}$. The amino acids sequence length of FADs ranged varied from 307 to 455 amino acids (aa). The predicted molecular weight (MW) of these proteins ranged from $32.48 \mathrm{kDa}$ (JrSLD2) to $52.06 \mathrm{kDa}(\mathrm{JrFAD} 7)$, and the theoretical isoelectric points (pI) ranged from 5.40 to 9.95 .

The length of JrFAD3-1 is $2402 \mathrm{bp}$, including 8 exons and 7 introns; the coding region is $1143 \mathrm{bp}$, encoding 380 amino acids.

\section{Analyses of the evolution, exon-intron structure and motif distribution of JrFAD family members}

Using MEGA 5.0 software with the maximum likelihood (ML) method, the walnut FADs protein sequences were constructed together with the Arabidopsis FADs protein sequences to build a phylogenetic tree (Fig. 1), indicating that the FAD gene families of $J$. regia and A. thaliana are similar. There are four main subfamilies: the SAD desaturase subfamily, $\Delta 7 / \Delta 9$ desaturase subfamily, $\Delta 12 /$ 
Table 1 Basic information of the FAD family in walnut

\begin{tabular}{|c|c|c|c|c|c|c|c|c|c|}
\hline Name & Locus ID & NCBI Reference & Gene length (bp) & Exon number & Intron number & CDS (bp) & Amino acid length (AA) & $\mathrm{Pl}$ & $\mathrm{MW}(\mathrm{KD})$ \\
\hline JrADS3 & LOC108998330 & XM_018974856.1 & 2497 & 5 & 4 & 1164 & 387 & 9.95 & 44.56 \\
\hline JrALD & LOC109021249 & XM_019003847.1 & 2055 & 1 & 0 & 1344 & 447 & 8.56 & 51.34 \\
\hline $\mathrm{JrDAL}$ & LOC109001694 & XM_018979084.1 & 4240 & 1 & 0 & 1149 & 382 & 8.78 & 44.11 \\
\hline JrDES1-1 & LOC109011517 & XM_018992767.1 & 2103 & 2 & 1 & 981 & 326 & 7.27 & 37.91 \\
\hline JrDES1-2 & LOC108990634 & XM_018964647.1 & 2623 & 2 & 1 & 978 & 325 & 7.83 & 37.74 \\
\hline JrFAD2-1 & LOC109021930 & XM_019004668.1 & 3197 & 1 & 0 & 1167 & 388 & 8.72 & 44.38 \\
\hline JrFAD2-2 & LOC109021930 & XM_019004667.1 & 3411 & 1 & 0 & 1167 & 388 & 8.72 & 44.38 \\
\hline JrFAD2-3 & LOC109011954 & XM_018993369.1 & 6737 & 1 & 0 & 1152 & 383 & 8.83 & 43.90 \\
\hline JrFAD2-4 & LOC109011954 & XM_018993367.1 & 4273 & 1 & 0 & 1152 & 383 & 8.83 & 43.90 \\
\hline JrFAD3-1 & LOC109002248 & XM_018979912.1 & 2402 & 8 & 7 & 1143 & 380 & 8.26 & 43.88 \\
\hline JrFAD3-2 & LOC108989905 & XM_018963672.1 & 2166 & 7 & 6 & 1053 & 350 & 7.79 & 40.51 \\
\hline JrFAD3-3 & LOC108989905 & XM_018963671.1 & 2166 & 8 & 7 & 1143 & 380 & 7.37 & 43.61 \\
\hline JrFAD3-4 & LOC108989903 & XM_018963670.1 & 2129 & 7 & 6 & 1077 & 358 & 7.80 & 41.43 \\
\hline JrFAD3-5 & LOC108989903 & XM_018963669.1 & 2128 & 8 & 7 & 1167 & 388 & 7.39 & 44.50 \\
\hline JrFAD4 & LOC108983937 & XM_018955731.1 & 1262 & 1 & 0 & 924 & 307 & 9.11 & 34.68 \\
\hline JrFAD6 & LOC108993197 & XM_018968019.1 & 4917 & 10 & 9 & 1329 & 442 & 9.18 & 51.38 \\
\hline JrFAD7 & LOC109007160 & XM_018986723.1 & 3038 & 8 & 7 & 1368 & 455 & 8.94 & 52.06 \\
\hline JrFAD8-1 & LOC108994930 & XM_018970364.1 & 2963 & 8 & 7 & 1368 & 455 & 8.98 & 51.77 \\
\hline JrFAD8-2 & LOC109015144 & XM_018997622.1 & 2965 & 8 & 7 & 1368 & 455 & 8.98 & 51.77 \\
\hline JrSAD-1 & LOC109005061 & XM_018983828.1 & 5401 & 3 & 2 & 1191 & 396 & 6.44 & 45.26 \\
\hline JrSAD-2 & LOC108984606 & XM_018956614.1 & 5286 & 3 & 2 & 1191 & 396 & 6.39 & 45.16 \\
\hline JrSAD-3 & LOC108984606 & XM_018956616.1 & 5039 & 2 & 1 & 1074 & 357 & 5.72 & 40.99 \\
\hline JrSAD-4 & LOC108984606 & XM_018956617.1 & 2456 & 2 & 1 & 999 & 332 & 5.40 & 38.05 \\
\hline JrSAD-5 & LOC108988959 & XM_018962389.1 & 2413 & 3 & 2 & 1176 & 391 & 7.72 & 44.43 \\
\hline JrSAD6 & LOC109012153 & XM_018993662.1 & 1549 & 2 & 1 & 1185 & 394 & 5.98 & 44.63 \\
\hline JrSAD-6 & LOC108989429 & XM_018963029.1 & 2075 & 3 & 2 & 1206 & 401 & 8.71 & 45.49 \\
\hline JrSAD-7 & LOC108988967 & XM_018962401.1 & 1981 & 3 & 2 & 1170 & 389 & 8.26 & 44.32 \\
\hline JrSAD-8 & LOC109018931 & XM_019001129.1 & 14,280 & 4 & 3 & 1002 & 333 & 6.16 & 38.08 \\
\hline JrSLD-1 & LOC109009165 & XM_018989549.1 & 1905 & 1 & 0 & 1344 & 447 & 6.64 & 32.64 \\
\hline JrSLD-2 & LOC109002707 & XM_018980564.1 & 1846 & 1 & 0 & 1344 & 447 & 6.42 & 32.48 \\
\hline
\end{tabular}

$\omega-3$ desaturase subfamily and "front-end" desaturase subfamily.

To date, SAD is the only subfamily of soluble enzymes in the FAD family, and the remaining types of fatty acid desaturases are membrane integrins $[25,26]$. There are 7 copies of the $S A D$ gene in Arabidopsis and 9 in walnut, and these genes are well clustered in the unified phylogenetic tree. In the $\Delta 12 / \omega-3$ desaturase subfamily, six branches of the walnut $\omega-6$ desaturase and two from Arabidopsis grouped together as $\Delta 12$ fatty acid desaturases. Three Arabidopsis FAD3 genes and eight annotated $\omega-3$ desaturase genes in walnuts together form the $\Delta 12 / \omega-3$ desaturase subfamily; among the five FAD3 genes of walnut, FAD3-1 was found to be far from the other four in evolutionary distance. Based on a comparison of gene expression levels during the development of walnut kernels (Fig. 2 and Additional file 1 Table S1), expression of $F A D 3$ was strongly detected in developing kernels; the other four genes were not expressed or expressed at very low levels, indicating that FAD3-1 is the key enzyme gene that catalyses the synthesis of linolenic acid in walnut kernels. A similar expression profile was reported for $F A D$ genes in olive, and siRNA was able to suppress the expression of other FADs but not FAD2-3 [27].

To further investigate the structural evolution of walnut FADs, we first analysed the exon-intron structure (Fig. 3). The FAD genes contain 1-9 introns except JrALD, JrSLD-1, JrSLD-2, JrDAL, JrFAD2-1, JrFAD2-2, JrFAD2-3, JrFAD2-4 and JrFAD4. Moreover, genes in the same subfamily have similar intron and exon structures. JrFAD6 differs from other $\Delta 12$ desaturase genes in that it has the largest number of introns and exons. Both 


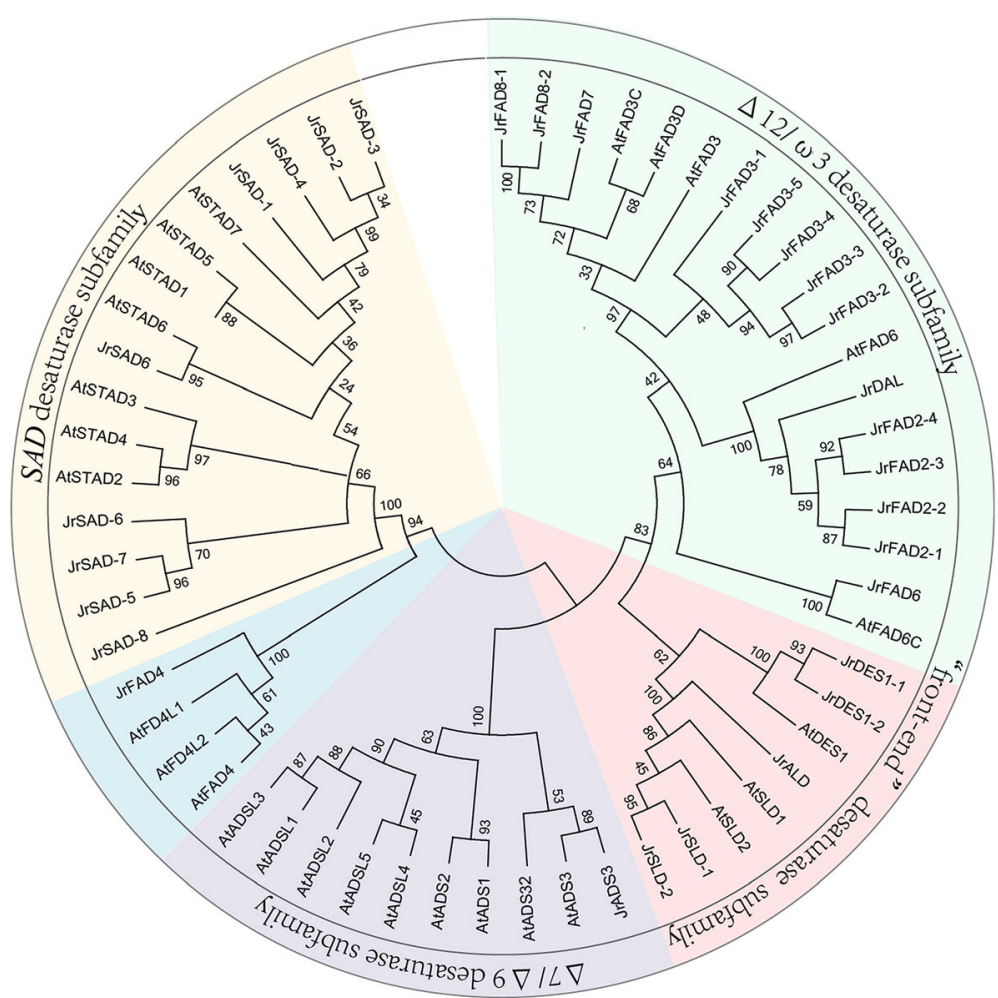

Fig. 1 Phylogenetic analysis of JrFADs (Juglans regia) and AtFADs (Arabidopsis thaliana) with a total of 57 protein sequences. MEGA 5.0 was used to construct the phylogenetic tree employing the maximum likelihood (ML) method. A total of 1000 bootstrap replications were carried out to indicate reliability

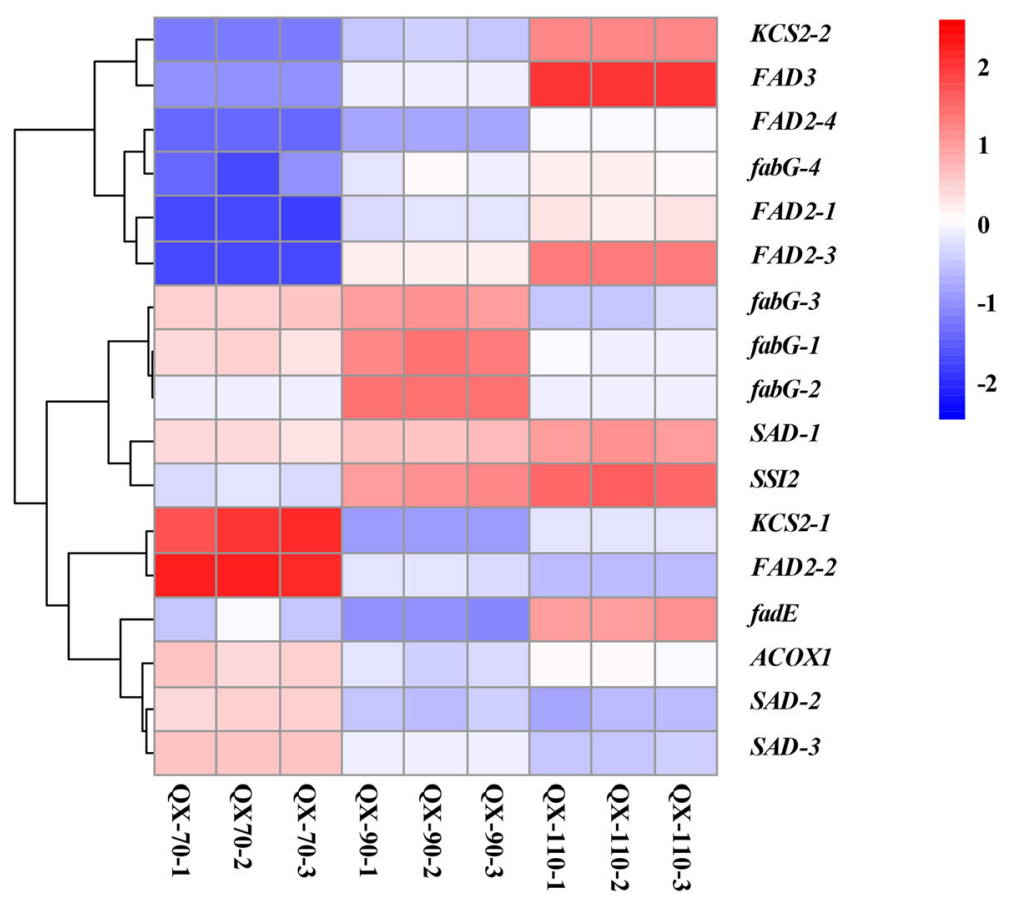

Fig. 2 Expression profilles (heatmaps) of genes associated with unsaturated fatty acid biosynthesis. Red indicates high gene expression level; white indicates medium level and blue indicates low level 


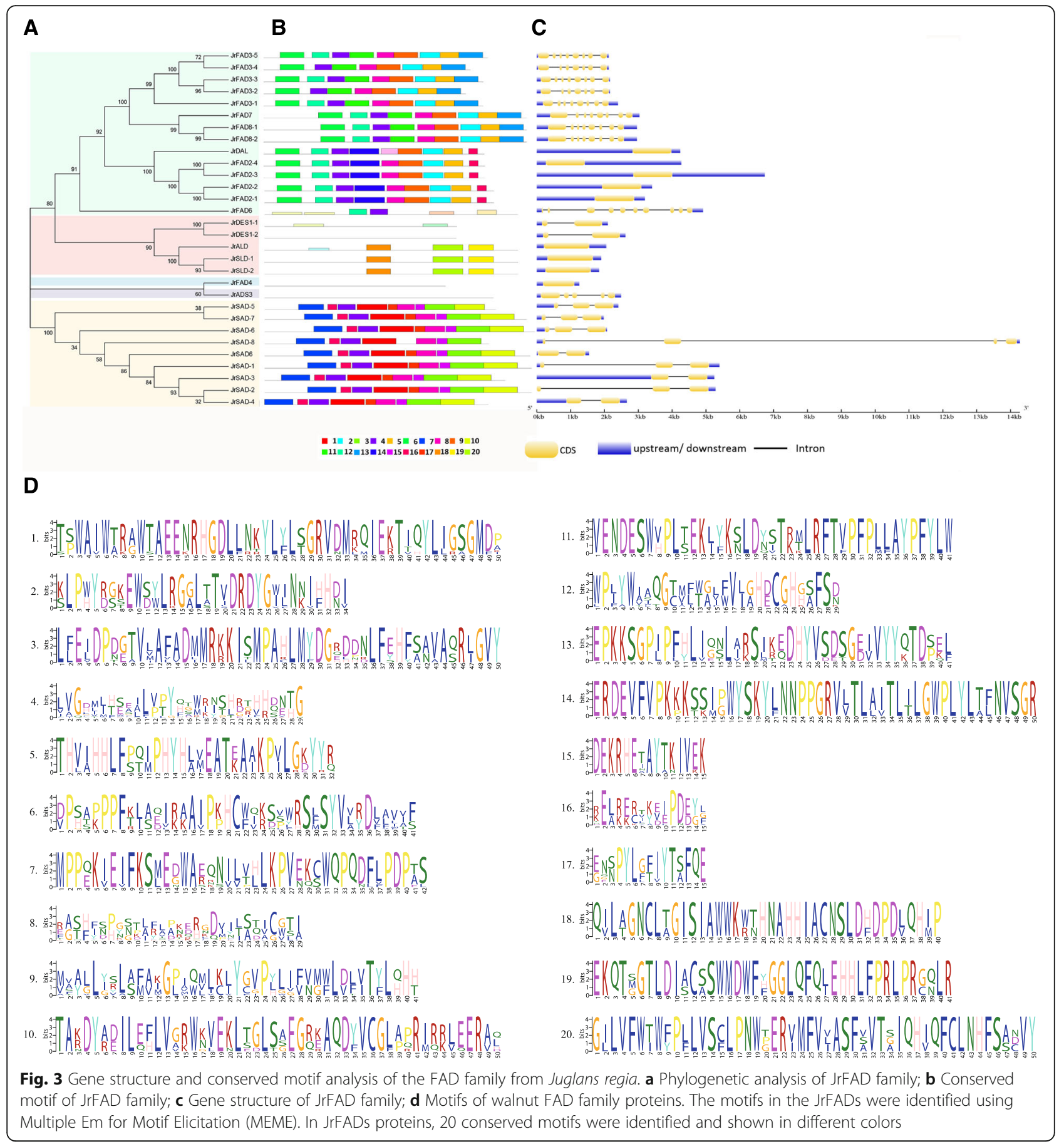

$J r D E S 1$ genes contain two exons, whereas JrALD and JrSLDs genes have only one exon. The number of exons in the SAD subfamily range between 2 and 4. JrSAD-8 contains 4 exons, the highest number; while JrSAD6, JrSAD-3 and JrSAD-4 contain 2 exons, and the remaining genes contain 3 exons (Fig. 3c).

Subsequently, we used MEME software to analyse the conservation of these protein sequences and structures (Fig. 3b). Most of the 20 conserved motifs (Fig. 3d) found belong to the typical transmembrane helix region and unknown functional complex region in fatty acid desaturases. Although no common conserved motifs were observed among the 30 members of the walnut FAD family, the distribution of conserved motifs was very similar within the same subfamily. In the SAD subfamily, all genes except JrSAD-8 contain conserved motifs $1,-3,-4,-7$ and -10 ; 
in the $\Delta 12 / \omega-3$ subfamily, the genes contain conserved motifs $2,-4,-5,-6,-8$ and -9 except JrFAD6.

\section{Expression of genes related to unsaturated fatty acid synthesis}

A heap map of our RNA-seq data highlighted differential expression of important metabolic pathways genes at kernel development stages, which showed the expression levels of lipid metabolism related genes were higher at late stage (Additional file 2 Fig. S1; Additional file 3 Table S2). The expression of genes related to unsaturated fatty acid biosynthesis and arachidonic acid metabolism peaked at 110 DAF based on the number of reads by transcriptome sequencing. The expression of genes related to alpha-linolenic acid metabolism first decreased and then increased, and reaching the maximum value at 110 DAF, based on the number of reads by transcriptome sequencing (Additional file 4 Fig. S2; Additional file 5 Table S3).

Seventeen genes enriched in the metabolic pathway of unsaturated fatty acid biosynthesis were selected (Fig. 2). KCS2 (encoding 3-ketoacyl-CoA thiolase), and fabG (encoding 3-oxoacyl-ACP reductase) are involved in carbon chain elongation; fadE (encoding acyl-ACP desaturase), SSI2 (encoding acyl-ACP desaturase), FAD2 (encoding fatty acid desaturase 2), FAD3-1(encoding fatty acid desaturase 3), $A C O X 1$ (encoding peroxisomal acyl-CoA oxidase) and $S A D$ (encoding stearoyl-ACP-desaturase) are involved in the desaturation process. The observed expression of 17 transcripts of these 8 genes can be roughly divided into two categories. On the one hand, the expression level was lower at 70 DAF but higher at 110 DAF. There were 7 transcripts, fabG-4, KCS2-2, fadE, FAD2-1, FAD2-3, FAD2-4 and FAD3-1, which were mainly involved in the biosynthesis of linoleic acid, linolenic acid and isounsaturated fatty acids. On the other hand, the expression levels were relatively high at 70 DAF and then gradually decreased. Ten transcripts, fabG-1, fabG-2, fabG-3, SAD-1, SAD-2, SAD-3, SSI2, $A C O X 1, K C S 2-1$ and $F A D 2-2$, mainly participate in the biosynthesis of oleic acid and linoleic acid. Expression of FAD3-1 increased rapidly during the period from 70 DAF to 110 DAF. The dehydrogenation of linoleic acid to $\alpha$ - linolenic acid began at $70 \mathrm{DAF}$, though the $\alpha$ linolenic acid content was almost zero, but genes encoding enzymes that catalyze the dehydrogenation of linoleic acid to linolenic acid were highly expressed at this stage. With the rapid increase in the expression of FAD3-1, the content of $\alpha$-linolenic acid in the kernel began to increase gradually.

Tissue-specific expression of $J r F A D$ family genes By semi-quantitative detection (Fig. 4), SLD-1 was found to be expressed in all 8 tissues, among which the

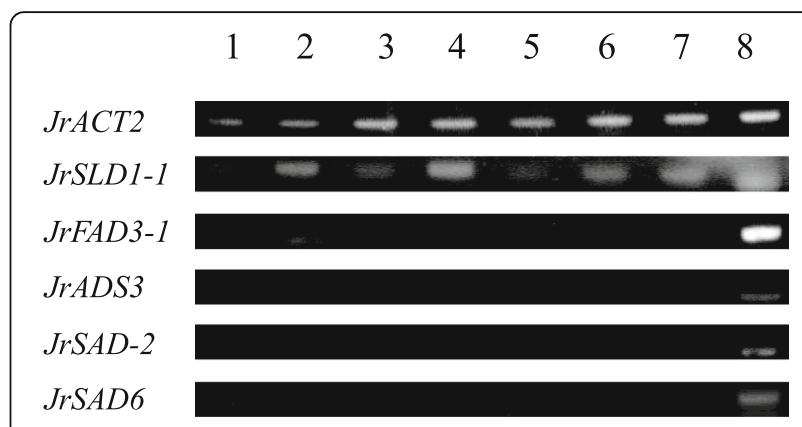

Fig. 4 Expression pattern of JrFAD genes in eight tissues/organs by RT-PCR. JrACT2 was used as an internal control. From left to right: pistils, catkin, young stem, older stem, young leaves, mature leaves, young embryo, and mature embryo

expression levels were higher in catkins, old branches, mature leaves and kernels. $D A L$ was most highly expressed in young and mature leaves. JrFAD3-1 was expressed in catkins, young leaves and kernels, and the highest expression level was observed in mature embryos. $A D S 3, S A D-2$ and $S A D 6$ were only expressed in mature embryos. It can be preliminarily concluded that the FAD family is characterized by the desaturase subfamily and that the $\Delta 7 / \Delta 9$ desaturase subfamily is specifically expressed in the embryo but that the "front-end" desaturase subfamily is expressed in all tissues. The $\Delta 12 / \omega-3$ desaturase subfamily is highly expressed in mature embryos. However, the determination of more specific expression patterns still requires further research and verification.

\section{Expression of JrFAD3-1 and accumulation of a-linolenic} acid in kernels at different developmental stages

The relative expression of JrFAD3-1 increased slowly at 70 DAF in the 'Qingxiang' kernel, increasing rapidly after 90 DAF and peaking at 100 DAF (Fig. 5). Then, its expression quickly decreased and gradually stabilized at

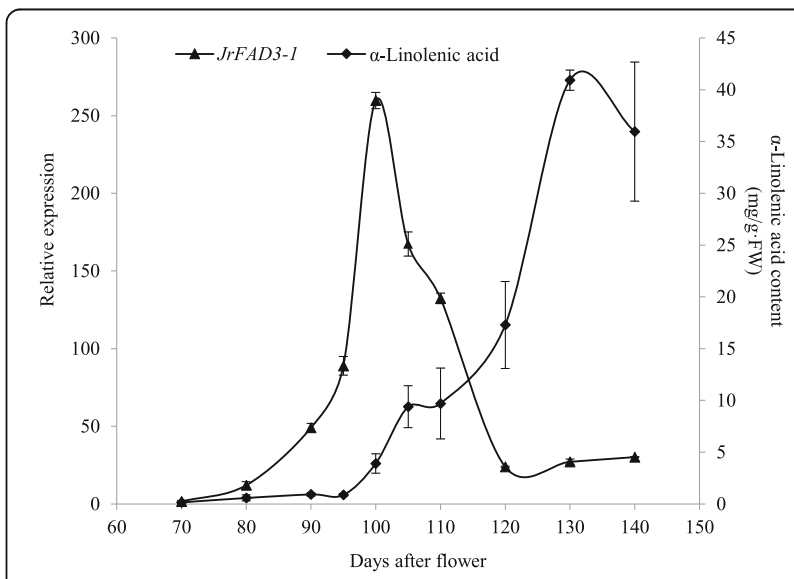

Fig. 5 Relative expression of JrFAD3-1 gene and a-Linolenic acid content in the kernels at different developmental stages 
a lower level after 120 DAF. We also detected the content of $\alpha$-linolenic acid in identical samples and found that it was maintained at a low level from 70 DAF to 95 DAF and gradually accumulated from 95 DAF to 120 DAF. The content increased rapidly from 120 DAF to $130 \mathrm{DAF}$ and peaked at $40.92 \mathrm{mg} / \mathrm{g}$, after which it decreased slightly (Fig. 5). Combining the two results revealed a 30-day difference between the peak of JrFAD3-1 gene expression and that of $\alpha$-linolenic acid content. Expression of JrFAD3-1 at 70-100 DAF and content of $\alpha-$ linolenic acid at 100-130 DAF was also analysed, showing a significant positive correlation between expression of JrFAD3-1 at 5 periods (70-100 DAF) and the content of $\alpha$-linolenic acid at 5 periods (100-130 DAF), with a correlation coefficient of 0.991 . Normally, the kernel water content decreased during the late fruit development [1]. Therefore, it is possible that the decrease in JrFAD3-1 expression and the decrease in water content in the late stage are factors contributing to the increase in the $\alpha$-linolenic acid content.

\section{Multiple sequence alignment of FAD3 proteins}

Multiple sequence alignment of the FAD3 proteins among 32 species was performed using MEGA 5.0. The results showed that FAD3 proteins were conserved between monocotyledonous and dicotyledonous plants and yeast. Additionally, conserved domain prediction was performed by a database search of the Pfam protein domain family, and all FAD3 proteins of the 32 species contain the fatty acid desaturase motif. Through comparative analysis, 62 completely conserved sites in the 33 protein sequences were found (Additional file 6 Fig. S3). It can be further confirmed that the primary structure of the JrFAD3-1 protein is closely related to the content of $\alpha$-linolenic acid.

\section{Phylogenetic evolution of FAD3 proteins}

Using MEGA 5.0, the retrieved homologous proteins were assessed along with the JrFAD3-1 protein and the reported sequences from the 32 species mentioned above. Subsequently, we used MEME software to analyse conservation among these protein sequences. Most of the 10 conserved motifs (Fig. 6) belong to the typical transmembrane helix region and unknown functional complex region in fatty acid desaturases. Three common conserved motifs (motif 2 , motif 3 and motif 8 ) were found among the 33 FAD3 proteins of the 32 species, but the distribution of conserved motifs was very similar within the same branch. The 32 species are divided into 2 branches (Fig. 7). Candida tropicalis is in one group with only 3 motifs (motif 2, motif 3, motif 8), and the other 31 plants are in another group. Of the latter, the 31 species are divided into 2 branches. Paeonia suffruticosa is a kind of flower or oil crop, and it grouped alone; the other plants clustered in another group. Picea abies and Olea europaea also group together though they are evolutionarily far, and the other comprise another group. The remaining 28 species are divided into 2 groups, 2 monocots (Oryza sativa and Triticum aestivum) and 26 dicots (e.g., Glycine max, Phaseolus lunatus, J. regia). Except for Crepis alpina, Physaria fendleri, and O. sativa, all FADs of 28 higher plants contain conserved motifs $1-10$; the FAD3 of $O$. sativa contains 8 conserved motifs except motif 6 and motif 9. JrFAD3-1 is closely related to homologous genes of Betula pendula (85.75\%) and Corylus heterophylla (84.92\%). B. pendula, C. heterophylla and $J$. regia all belong to the order Fagales, which further indicates that the conserved structure of FADs is consistent with classical plant taxonomy.

\section{Discussion}

To date, the FAD gene family has been identified and characterized in many plants, including 17 FADs in Arabidopsis [21], 29 in soybean [28], 31 in peanut [29], and 41 in Raymond cotton [30]. FAD genes have also been isolated from land cotton [31], sesame [32], sunflower [33] and other plants. In this study, 24 FAD family members were identified and analysed based on protein properties. In addition, a similar genetic structure was observed for each small branch, with high subfamily conservation. For example, the $\Delta 12 / \omega-3$ desaturase subfamily contains 7-8 exons, consistent with Arabidopsis [21] and Carya cathayensis [34]. There are 2-3 exons in SAD subfamily genes, consistent with peanut [29]. Studies have shown that the origin of introns is ancient and that insertion of introns is the result of exon

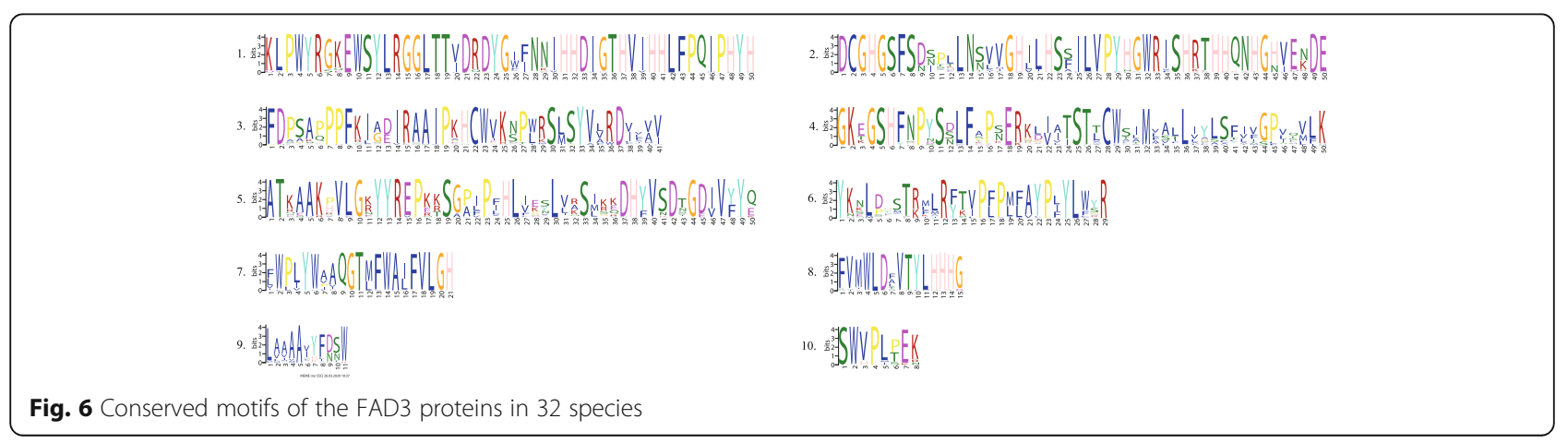



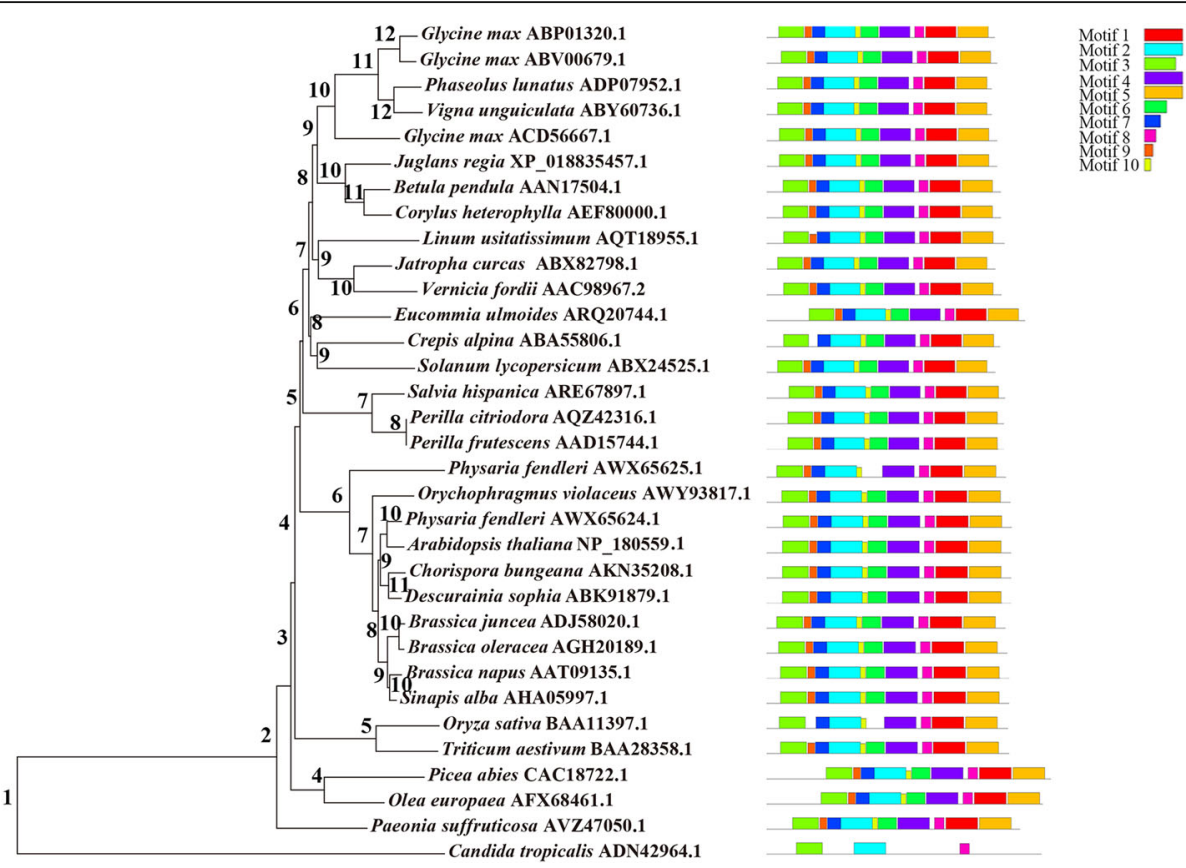

Fig. 7 Phylogeny conserved motif of FAD3 proteins in 32 species. Number represents branch series. The motifs in the FAD3 proteins were identified using MEME. Ten conserved motifs were identified of 32 species and shown in different colors

rearrangement, with an important role in gene evolution $[35,36]$. By analysing gene sequences, the $\operatorname{JrFAD}$ family was found to be highly conserved, though there are large differences between subfamilies. The FAD family of walnut and Arabidopsis is composed of four major groups: the SAD desaturase subfamily, the $\Delta 7 / \Delta 9$ desaturase subfamily, the $\Delta 12 / \omega-3$ desaturase subfamily and the "frontend" desaturase subfamily [21]. There are large differences in the copy number of genes in each subfamily. The $\Delta 9$ desaturase subfamily in Arabidopsis has the most common gene copy number of 9 [21], whereas the gene copy number is only one in walnut. Similar to the results for walnut, $\Delta 9$ desaturase was not identified in the genome of soybean; instead, $S A D$ [28], which also generates the first unsaturated bond as dose $\Delta 9$ desaturase, was detected. This suggests that $\Delta 9$ desaturase, which is widely distributed among organisms, is replaced by the SAD subfamily in higher plant, with similar functions and greater copy numbers, which may be related to the evolution of the $S A D$ gene family in plants [21]. The number of $\Delta 12 / \omega-3$ desaturase subfamily members is also higher in walnut than in Arabidopsis, probably because these genes have not undergone related genome-wide replication during evolution.

There is accumulating evidence that the linolenic acid content is closely related to the FAD3 gene [37-40]. In Arabidopsis seeds, specific overexpression of endogenous $F A D 3$ increased the $\alpha$-linolenic acid content from 19 to $40 \%$ [41], and overexpression of FAD3 genes in tomato resulted in significantly increased $\alpha$-linolenic acid levels in the leaves and fruits of the transgenic plants [42]. Ni et al. [43] reported that the content of $\alpha$-linolenic acid in rapeseed increased when $B n F A D 3$ was overexpressed. Inhibition of tobacco FAD3 gene expression through RNAi technology significantly reduced the accumulation of $\alpha$-linolenic acid [44]. Liao et al. [45] suppressed the expression of FAD2, FAD3 and FATB in rapeseed by constructing a multi-gene interference vector containing the seed-specific promoter NapinA, increasing the oleic acid content by more than $16 \%$. The content of linolenic acid in walnut kernels was high, and 5 copies of JrFADs were identified in the genome-wide range.

Although 5 FAD3 copies were identified from the walnut genome, only JrFAD3-1 expression was found in the walnut kernel during development, with the peak expression at approximately 100 DAF. Although JrFAD3-1 is not a seed-specific gene, expression of this gene was significantly upregulated during seed development, showing a trend of increase and then decrease, with a tendency toward a decline as the seed matures, consistent with the findings of previous studies [46, 47]. This change may be related to the important role of FAD3 in the synthesis of unsaturated fatty acids in walnut [48]. There may be two reasons for the change in $\alpha$-linolenic acid content after 30 days in this study. First, a process in the cytoplasm may be involved. Proteins are involved in mRNA production and must be transported from the cytoplasm to the nucleus after translation; there is also a 
time lag in gene expression due to such processed as post-translational modification $[49,50]$. Second, the $\alpha-$ linolenic acid content is directly related to the amount of jasmonic acid produced; $\omega-3$ fatty acid dehydrogenase (FAD) catalyses the synthesis of $\alpha$-linolenic acid from linoleic acid [51]. JrFAD3-1 encodes $\Delta 15$ fatty acid desaturase in the seed, catalysing the conversion of linoleoyl CoA to $\alpha$-linoleyl CoA. $\alpha$-Linolenic acid is the substrate for jasmonic acid [52]. The protein content reached its highest level at $110 \mathrm{DAF}$, showing a positive correlation with the oil content [53]. It is also possible that the $\alpha$ linolenic acid content due to a lack of substrate in the early stage was not obvious early in embryo development in walnut. As the embryo matured, expression of JrFAD3-1 was downregulated, and the $\alpha$-linolenic acid content increased. Overall, desaturation gradually decreased after expression peaked, which may have been caused by the decrease in FAD3 activity and the consumption of $\alpha$-linoleic acid. In general, gene function can be explained comprehensively through positive and negative aspects, providing a theoretical basis for establishing a complete metabolic network.

\section{Conclusions}

This study described the FAD gene family of walnut at the genome level. Their gene structure, phylogenetic relationship, and tissue-specific expression patterns were presented in this study. A total number of 24 members of the $J r F A D$ gene family were identified and classified into four major subfamilis. JrFAD3-1, a key gene in $\triangle 12 / \omega-3$ desaturase subfamily, was obtained based on transcriptome data, and its expression was analysed. The function of JrFAD3-1 was also characterized based on the deduced phylogeny. The result predicates that $J r F A D 3-1$ may play a key role in the biosynthesis of polyunsaturated fatty acids. This study lays the foundation for further functional elucidation of JrFAD genes in walnut.

\section{Methods}

\section{Plant materials}

The material used in this study was collected from the Experimental Field of Hebei Agricultural University in 2016. A walnut cultivar of 'Qingxiang' in full fruitbearing period was selected as the test material.

The samples used for transcriptome sequencing were 'Qingxiang' kernels collected 70 (QX-70), 90 (QX-90) and 110 (QX-110) DAF. Nine samples of walnut kernels at each stage were collected from 'Qingxiang' trees. Several grams of different tissues from each plant were frozen in liquid nitrogen and stored at $-80^{\circ} \mathrm{C}$.

JrFAD3-1 gene expression analysis was conducted with 'Qingxiang' samples from 10 different developmental stages (70 DAF, 80 DAF, 90 DAF, 95 DAF, 100 DAF,
105 DAF, 110 DAF, 120 DAF, 130 DAF and 140 DAF), and 8 different tissues: pistils, catkins, young branches (bark), old branches (bark), young leaves, mature leaves, young embryos and mature embryos. After the fruits were collected, the embryos were quickly removed by peeling and immersed in liquid nitrogen, and stored at $-80{ }^{\circ} \mathrm{C}$ for RNA extraction, expression analysis and determination of fatty acid content.

The kits used for RNA extraction and DNA extraction were from Tiangen (product numbers: DP441 and DP350 respectively). The reagents used for reverse transcription, PCR and real-time PCR were from Takara (product numbers: RR047A, RR901A and RR820A, respectively).

\section{Cloning of the JrFAD3-1 gene}

The JrFAD3-1 gene was amplified from kernels collected 100 DAF. The JrFAD3-1 partial gene sequence was identified from walnut genome data, and a homology-based cloning method was used to isolate the JrFAD3-1 coding sequence (CDS) from 'Qingxiang' walnut according to the gene sequences of soybeans and castor beans. Premier 5.0 software was used to design specific primers as follows: JrFAD3-1-F: 5'- GAGAAAGGAGGAGAGAATGC-3', JrFAD3-1-R: 5'- GATAGCCTTGCT. CTTCAAA-3'.

\section{Identification of FAD gene family and bioinformatics analysis}

The hidden Markov model was constructed using HMMER 3.1b software and the Arabidopsis FAD family protein sequence (Additional file 7 Table S4) in the UniProt database and the protein sequences of walnut were downloaded from GenBank (accession GCF_001411555.1). The conserved domains of the proteins were analysed using the Pfam protein family database (http://pfam.org/) [54] of the European Institute of Bioinformatics, using the website GSDS (Gene Structure Display Server: http://gsds. cbi.pku.edu.cn/) [55] and MEME (http://meme-suite.org/) pair for the walnut FAD family. The phylogenetic tree of JrFAD and AtFAD gene family was constructed using the maximum likelihood method in MEGA 5.0 software with a bootstrap value of 1000 . The phylogenetic tree JrFAD and 31 other species were built using the maximum likelihood method in MEGA 5.0 software with a bootstrap value of 1000 .

\section{Analysis of transcriptome sequencing data}

The predicted coding transcripts were aligned with the GenBank NR (https://www.ncbi.nlm.nih.gov/nucleotide/), Swiss-Prot (https://www.uniprot.org/), InterPro (http:// www.ebi.ac.uk/interpro/), GO (http://geneontology.org), and KEGG databases (https://www.kegg.jp/) for functional annotation (Additional file 8). 
Table 2 Primers sequences of JrFADs and JrACT2 for qRT-PCR

\begin{tabular}{lll}
\hline Name & Sequence $\left(5^{\prime}-3^{\prime}\right)$ & \\
\cline { 2 - 3 } JrACT2 & F: TCCACCATGTTCCTGGTAT; & R: ACCTCCCAATCCAGACACTG \\
\hline JrSLD-1 & F: ACTTGGTTGGGAGGCTGTC; & R:GTCCTAACAACCCCAAGC \\
JrADS3 & F: TTCCTTGTGTGGGGATGGGG; & R: TGGTGTAAAAGGCTTCTTTGGATTG \\
JrSAD-2 & F: TITTCCCCTTCTCAACCTCCAA; & R: CCAGCTAAACTTAACGCCGC \\
JrSAD6 & F: AAGCTGGAGGATTGACGGG; \\
JrFAD3-1 & F: ATCCACCATGATATCGGCACC; & R: GAAGGGGAATTGGCCCAGA \\
\hline
\end{tabular}

\section{Tissue-specific expression in the JrFAD family}

For RNA isolation experiments, all samples were immediately frozen in liquid nitrogen and extracted using a Tiangen Plant RNA Extraction Kit according to the manufacturer's protocol. First-strand cDNA was synthesized with a Tiangen Inverse Transcription Kit. The gene-specific primers used for RT-PCR and qRT-PCR were designed using NCBI (Table 2). Five genes belonging to four subfamilies were selected to analyse the expression of the FAD families in different tissues.

The PCR procedure was as follows: $94{ }^{\circ} \mathrm{C}$ for $5 \mathrm{~min}$; 33 cycles of $94{ }^{\circ} \mathrm{C}$ for $30 \mathrm{~s}, 54{ }^{\circ} \mathrm{C}$ for $30 \mathrm{~s}$, and $72^{\circ} \mathrm{C}$ for 1 min; and $72{ }^{\circ} \mathrm{C}$ for $10 \mathrm{~min}$.

\section{Gene expression analysis by quantitative RT-PCR}

qRT-PCR experiments were performed using an FX96 Touch $^{\text {Tu }}$ Real-Time PCR Detection System (Bio-Rad) with a SYBR Green I Master Kit (Tiangen). JrACT2 (NCBI Reference: XM_018972062.1) was used as the reference gene to calculate relative fold differences based on the comparative cycle threshold $\left(2^{-\Delta \Delta c t}\right)$. The PCR mixture $(20 \mu \mathrm{L})$ contained $10 \mu \mathrm{L}$ SYBR-Green PCR Master Mix (Tiangen), $0.2 \mu \mathrm{M}$ each primer, $100 \mathrm{ng}$ cDNA template, and nuclease-free water. The PCR procedure was as follows: $95^{\circ} \mathrm{C}$ for $5 \mathrm{~min} ; 30$ cycles of $94^{\circ} \mathrm{C}$ for 10 $\mathrm{s}, 60^{\circ} \mathrm{C}$ for $30 \mathrm{~s}$, and $72{ }^{\circ} \mathrm{C}$ for $30 \mathrm{~s}$; followed by a final extension at $72{ }^{\circ} \mathrm{C}$ for $3 \mathrm{~min}$.

\section{Determination of fatty acid content}

The determination of unsaturated fatty acids referred to Bai [56].

\section{Statistical analysis}

Data were analysed using SPSS 17.0 software.

\section{Supplementary information}

Supplementary information accompanies this paper at https://doi.org/10. 1186/s12864-020-6692-z

Additional file 1 : Table S1 Expression data of genes associated with unsaturated fatty acid biosynthesis.

Additional file 2 : Fig. S1 Clustering analysis (heatmap) of annotations for metabolic pathways.
Additional file $\mathbf{3}$ : Table S2 Expression data of the key metabolic pathways genes at kernel development stages.

Additional file 4 : Fig. S2 Expression of fatty acid biosynthesis metabolic pathway-related genes at different development stages.

Additional file $\mathbf{5}$ : Table $\mathbf{S 3}$ Expression of genes related to unsaturated fatty acid biosynthesis and arachidonic acid metabolism.

Additional file $\mathbf{6}$ : Fig. $\mathbf{3 3}$ Multiple conserved sequence alignment of FAD3 proteins in 32 species.

Additional file $\mathbf{7}$ : Table $\mathbf{S 4}$ Information of the FAD family in Arabidopsis thaliana.

Additional file 8. Annotation genes sequences of transcriptome data.

\section{Abbreviations}

FAD2: Microsomal $\Delta 12$ desaturase; FAD3: Microsomal $\omega$-3 desaturase: FAD4: Trans $\Delta 3$ desaturase; FAD5: $\Delta 7$ desaturase; FAD6: Plastidial $\Delta 12$ desaturase; FAD7: Plastidial $\omega-3$ desaturase; FAD8: Plastidial $\omega 3$ desaturase; ADS: $\triangle 9$ desaturase; SAD: Stearyl acyl-carrier-protein desaturase; ALD: Acyllipid (9-3)-desaturase; SCD: Stearoyl-CoA-desaturase; ACCase: Acetyl coenzyme A carboxylase; KCS2: 3-ketoacyl-CoA thiolase; fabG: 3-oxoacyl-ACP reductase; fadE: Acyl-CoA dehydrogenase; ACP: Acyl-carrier protein;

DAL: $\Delta 12$-acyl-lipid-desaturase; DES1: Sphingolipid $\Delta 4$-desaturase; SSI: AcylACP desaturase; ACOX: Peroxisomal acyl-CoA oxidase; SLD: $\Delta 8$-desaturase; Jr: Juglans regia

\section{Acknowledgements}

We would also like to thank Dr. Jin Zhao for her valuable suggestions on the data analysis and manuscript.

\section{Authors' contributions}

$\mathrm{SZ}, \mathrm{HW}$ and ZZ designed the research. KL and SZ performed the experiments, analyzed the data and wrote the paper. SW participated in the data analysis. $\mathrm{KL}$ and $\mathrm{SZ}$ are equal contributors. All authors read and approved the final the manuscript.

\section{Funding}

This work was supported by the Natural Science Foundation of Hebei Province (C2019204270), the Science and Technology Research Project of the Universities of Hebei Province (QN2019159), and Research Foundations for Returned Scholars from Overseas of the Human Resources Dept. of Hebei Province (C20190345). Funds were used for the design of the study, sample collection, analysis or interpretation of data and in writing the manuscript.

Availability of data and materials

All data and materials are presented in the main paper and additional file. In addition, the whole protein sequences of FADs in Arabidopsis were retrieved from TAIR databases. The CDS and genome sequences of FADs in walnut were retrieved from the whole walnut genome database (accession GCF_001411555.1) in NCBI.

\section{Ethics approval and consent to participate}

Walnut is one of widespread fruit trees in China, and it is not an endangered species. The walnut trees were from the Experimental Field of Hebei Agricultural University. No specific permits are required for sample collection on walnut. 


\section{Consent for publication}

Not applicable.

\section{Competing interests}

The authors declare that they have no competing interests.

\section{Author details}

'Mountainous Area Research Institute of Hebei Province, Hebei Agricultural University, Baoding 071001, China. ${ }^{2}$ College of Life Sciences, Hebei Agricultural University, Baoding 071001, China. ${ }^{3}$ Research Center for Agricultural Engineering Technology of Mountain District of Hebei, Baoding 071001, China. ${ }^{4}$ National Engineering Research Center for Agriculture in Northern Mountainous Areas, Baoding 071001, China.

Received: 7 November 2019 Accepted: 24 March 2020

Published online: 15 April 2020

\section{References}

1. Zhang ZH, Pei D. Walnut. 1st ed. Beijing: China Agricultural Press; 2018.

2. Wang XY, Zhang ZH, Li QY, Zhao HR, Zhao YP. Analysis of fatty acids composition and content in walnut varieties. Acta Nutri Sini. 2004;26(6): 499-501.

3. Zhao SG, Zhao YP, Wang HX, Zhang ZH. Physieal/chemieal characeteirsties and fatty acid composition of walnut oil. J Chin Cereal Oil Ass. 2008;23(2): 85-8.

4. Anderson KJ, Teuber SS, Gobeille A, Cremin P, Waterhouse AL, Steinberg FM. Walnut polyphenolics inhibit in vitro human plasma and LDL oxidation. J Nutr. 2001;131(11):2837-42.

5. Vingrys AJ, Armitage JA, Weisinger HS, Bui BV, Sinclair AJ, Weisinger RS. The role of omega-3 polyunsaturated fatty acids in retinal function. In: Mostofsky DI, Yehuda S, Salem N, editors. Fatty acids: physiological and Behavioural functions. New Jersey: Humana Press; 2001. p. 193-217.

6. Lu YD, Chi XY, Li ZX, Yang QL, Li FC, Liu SF, Gan QH, Qin S. Isolation and characterization of a stress- dependent plastidial $\Delta^{12}$-fatty acid desaturase from the antarctic microalga chlorella vulgaris NJ-7. Lipids. 2010;45(2):179-87.

7. Xue Y, Chen B, Win AN, Fu C, Lian J, Liu X, Wang R, Zhang X, Chai Y. Omega-3 fatty acid desaturase gene family from two $\omega-3$ sources, Salvia hispanica and Perilla frutescens: cloning, characterization and expression. PLoS One. 2018;13(1):e0191432.

8. Majou D. Evolution of the human brain: the key roles of DHA ( $\omega-3$ fatty acid) and $\Delta 6$-desaturase gene. OCL. 2018;25(4):A401.

9. Miller CN, Polasek LK, Oliveira AC, Frost CJ, Maniscalco JM. Milk fatty acid composition of perinatal and foraging steller sea lions (Eumetopias jubatus): examination from pup stomachs. Can J Zool. 2018:96(2):153-62.

10. Shanklin J, Cahoon EB. Desaturation and related modifications of fatty acids. Annu Rev Plant Physiol Plant Mol Biol. 1998;49:611-41.

11. Rawlings BJ. Biosynthesis of fatty acids and related metabolites. Nat Prod Rep. 1998;15(3):275-308.

12. Ntambi JM, Buhrow SA, Kaestner KH, Christy RJ, Sibley E, Kelly TJ, Lane MD. Differentiation-induced gene expression in 3T3-L1 preadipocytes. Characterization of a differentially expressed gene encoding stearoyl-CoA desaturase. J Biol Chem. 1988:263(33):17291-300.

13. Shanklin J, Somerville C. Stearoyl-acyl-carrier-protein desaturase from higher plants is structurally unrelated to the animal and fungal homologs. Proc Natl Acad Sci U S A. 1991:88(6):2510-4.

14. Kachroo A, Shanklin J, Whittle E, Lapchyk L, Hildebrand D, Kachroo P. The Arabidopsis stearoyl-acyl carrier protein-desaturase family and the contribution of leaf isoforms to oleic acid synthesis. Plant Mol Biol. 2007; 63(2):257-71.

15. Chen D, Yu Y, Yue C, Wang PJ, Chen J, Chen GX, Ye NX. Cloning and expression analysis of $\Delta 12$-fatty acid desaturase in tea plants. J Tea Sci. 2017;37(6):541-50

16. Gibson S, Arondel V, Iba K, Somerville C. Cloning of a temperature-regulated gene encoding a chloroplast $\omega-3$ desaturase from Arabidopsis thaliana. Plant Physiol. 1994;106(4):1615-21.

17. Mcconn M, Hugly S, Browse J. Somerville Chris. A mutation at the fad8 locus of Arabidopsis identifies a second chloroplast $\omega-3$ desaturase. Plant Physiol. 1994;106(4):1609-14.

18. Iba K, Gibson S, Nishiuchi T, Fuse T, Nishimura M, Arondel V, Hugly S, Somerville C. A gene encoding a chloroplast $\omega-3$ fatty acid desaturase complements alterations in fatty acid desaturation and chloroplast copy number of the fad7 mutant of Arabidopsis thaliana. J Biol Chem. 1993; 268(32):24099-105.

19. Hernandez ML, Sicardo MD, Martinezrivas JM. Differential contribution of endoplasmic reticulum and chloroplast $\omega-3$ fatty acid desaturase genes to the linolenic acid content of olive (Olea europaea) fruit. Plant Cell Physiol. 2016;57(1):138-51.

20. Los DA, Murata N. Structure and expression of fatty acid desaturases. Biochim Biophys Acta. 1998;1394(1):3-15.

21. Arondel V, Lemieux B, Hwang I, Gibson S, Goodman H, Somerville C. Mapbased cloning of a gene controlling omega-3 desaturation in Arabidopsis. Science. 1992;258(5086):1353-5.

22. Cui YP, Liu ZJ, Zhao YP, Wang YM, Huang Y, Li L, Wu H, Xu SX, Hua JP. Overexpression of heteromeric GhACCase subunits enhanced oil accumulation in upland cotton. Plant Mol Biol Rep. 2017;35(2):287-97.

23. Voellcer TA, Hayes TR, Cranmer AC. Genetic engineering of a quantitative trait: metabolic and genetic parameters influencing the accumulation of laurate in rapeseed. Plant J. 1996:9(2):229-41.

24. Roesler K, Shintani D, Savage L. Targeting of the Arabidopsis homomeric acetyl-coenzyme a carboxylase to plastids of rapeseeds. Plant Physiol. 1997; 113(1):75-81.

25. Xiao LJ, Zhang Y, Wu T, Ning DL, Mao YL. Fatty acid content and nutritional evaluation of Yunnan purple kernel walnut. China Oils and Fats. 2014:39(9):94-7.

26. Li WB, Lei F, Song W, Luo J, Bi WS, Liu Y, Xu LX, Zhao X. Accumulation dynamics of fatty acid in soybean seed and screening of candidate gene related to linolenic acid metabolism. J Northeast Agri Univ. 2017;48(11):1-8.

27. Unver T, Wu ZY, Sterck L, Turktas M, Lohaus R, Li Z, Yang M, He L, Deng TQ, Escalante FJ, Llorens C, Roig FJ, Parmaksiz I, Dundar E, Xie FL, Zhang BH, Ipek A Uranbey S, Erayman M, Ihan E, Badad O, Ghazal H, Lightfoot DA, Kasarla P. Colantonio V, Tombuloglu H, Hernandez P, Mete N, Cetin O, Van MM, Yang H, Gao Q, Dorado G, Vande P. Genome of wild olive and the evolution of oil biosynthesis. Proc Natl Acad Sci U S A. 2017;114(44):201708621.

28. Chi XY, Yang QL, Lu YD, Wang JY, Zhang QF, Pan LJ, Chen MN, He YN, Yu SL. Genome-wide analysis of fatty acid desaturases in soybean (Glycine max). Plant Mol Biol Rep. 2011;29(4):769-83.

29. Ruan J Shan L, Li XG, Guo F, Meng Jل Wan SB, Peng ZY Genome-wide identification and expression pattern analysis of peanut FAD gene family. Shandong Agri Sci. 2018:50(6):1-9.

30. Feng J, Dong Y, Liu W, He Q, Daud MK, Chen J, Zhu S. Genome-wide identification of membrane-bound fatty acid desaturase genes in Gossypium hirsutum and their expressions during abiotic stress. Sci Rep. 2017;1(7):1-12.

31. Pirtle IL, Kongcharoensuntorn W, Nampaisansuk M, Knesek JE, Chapman KD, Pirtle RM. Molecular cloning and functional expression of the gene for a cotton $\Delta 12$-fatty acid desaturase (FAD2). Biochim Biophys Acta. 2001;1522(2):122-9.

32. Jin UH, Lee JW, Chung YS, Lee JH, Yi YB, Kim YK. Characterization and temporal expression of a $\omega-6$ fatty acid desaturase cDNA from sesame (Sesamum indicum L.) seeds. Plant Sci. 2001:161(5):935-41.

33. Martínezrivas JM, Sperling P, Lühs W, Heinz E. Spatial and temporal regulation of three different microsomal oleate desaturase genes (FAD2) from normal-type and high-oleic varieties of sunflower (Helianthus annuus L.). Mol Breeding. 2001:8(2):159-68.

34. Huang CY, Huang YJ, Wu JF, Huang R, Luan YM, Zhang SM, Wang ZJ, Zhang QX, Huang JQ. SAD and FAD genes regulate the ratio of unsaturated fatty acid components in Carya cathayensis. Acta Hortic Sin. 2018;45(2):250-60.

35. Cavaliersmith T. Intron phylogeny:a new hypothesis. Trends Genet. 1991; 7(5):145-8.

36. Rzhetsky A, Ayala FJ, Hsu LC, Chang C, Yoshida A. Exon/intronstructure of aldehyde dehydrogznase genes supports the "introns-late" theory. Proc Natl Acad Sci U S A. 1997;94(13):6820-5.

37. Hita WD, Carlson TJ, Booth JR, Kinney AJ, Stecca KL, Yada NS. Cloning of a higher-plant plastid $\omega-6$ fatty acid desaturase cDNA and its expression in a Cyanobacterium. Plant Physiol. 1994;105(2):634-41.

38. Banilas G, Moressis A, Nikoloudakis N, Hatzopoulos P. Spatial and temporal expressions of two distinct oleate desaturases from olive (Olea europaea L.). Plant Sci. 2005;168(2):547-55

39. Teixeira MC, Coelho N, Olsson ME, Brodelius PE, Carvalho IS, Brodelius M. Molecular cloning and expression analysis of three $\omega-6$ desaturase genes from purslane (Portulaca oleracea L). Biotechnol Lett. 2009:31(7):1089-101.

40. Mikkilineni $V$, Rocheford TR. Sequence variation and genomic organization of fatty acid desaturase-2 (fad2) and fatty acid desaturase-6 (fad6) cDNAs in maize. Theor Appl Genet. 2003;106(7):1326-32. 
41. Puttick D, Dauk M, Lozinsky S, Smith MA. Overexpression of a FAD3 desaturase increases synthesis of a polymethylene-interrupted dienoic fatty acid in seeds of Arabidopsis thaliana L. Lipids. 2009;44(8):753-7.

42. Domínguez T, Hernández ML, Pennycooke JC, Jiménez P, Martínez-Rivas JM, Carlos S, Eric JS, José JS, Maite S. Increasing $\omega$-3 desaturase expression in tomato results in altered aroma profile and enhanced resistance to cold stress. Plant Physiol. 2010;153(2):655-65.

43. Ni XF, Zhao CZ, Li H, Zhang M. Construction of TmFAD2 and BnFAD3 double-gene expression vector and fatty acid analysis of transgenic plants. Acta Agric Boreali-Occident Sin. 2014;23(10):112-9.

44. Murakami Y, Tsuyama M, Kobayashi Y, Kodama H, Iba K. Trienoic fatty acids and plant tolerance of high temperature. Science. 2000;287(5452):476-9.

45. Liao XJ, Wang XJ, Wang B, Liu AZ. Cloning of the promoter of the key enzyme gene PVFAD3 from Sacha Inchi and its transcriptional activation analyses. Plant Physiol J. 2017;53(1):79-88.

46. Wang SY, Han B, Zhou SM, Xu J. Expression of functional genes related to fatty acid synthesis of oil flax. Chinese J of Oil Crop Sci. 2016;38(6):771-7.

47. Banik M, Duguid S, Cloutier S. Transcript profiling and gene characterization of three fatty acid desaturase genes in high, moderate, and low linolenic acid genotypes of flax (Linum usitatissimum L.) and their role in linolenic acid accumulation. Genome. 2011;54(6):471-83.

48. Vrinten P, Hu Z, Munchinsky MA, Qiu RX. Two fad3 desaturase genes control the level of linolenic acid in flax seed. Plant Physiol. 2005;139(1):79-87.

49. He Y, Zeng J, Wu M, Zhang CK, Zhang Y. Stability for discrete-time genetic regulatory network with time-verying interval delays. Control Theor Appl. 2012;29(11):1465-70

50. Pradet-Balade B, Boulmé F, Beug H, Müllner EW, Garcia-Sanz JA. Translation control: bridging the gap between genomics and proteomics? Trends Biochem Sci. 2001;26(4):225-9.

51. Makandar R, Nalam V, Chaturvedi R, Jeannotte R, Sparks AA, Shah J. Involvement of salicylate and jasmonate signaling pathways in Arabidopsis interaction with Fusarium graminearum. Mol Plant Microbe In. 2010;23(7):861-70.

52. Suza WP, Avila CA, Carruthers K, Kulkarni S, Goggin FL, Lorence A. Exploring the impact of wounding and jasmonates on ascorbate metabolism. Plant Physiol Bioch. 2010;48(5):337-50.

53. Chen H, Pan CD, Wang B, Hu Y, Xiao ZZ, He M. The relationship among nutrients' accumulation and dynamic changes of fatty acids in seed development of walnut. J Hebei Agri Univ. 2016;39(1):57-62.

54. Finn RD, Coggill P, Eberhardt RY, Eberhardt RY, Eddy SR, Mistry J, Mitchell AL, Potter SC, Punta M, Qureshi M, Sangrador-Vegas A, Salazar GA, Tate J, Bateman A. The Pfam protein families database: towards a more sustainable future. Nucleic Acids Res. 2016;44(Database issue):D279-85.

55. Guo A, Zhu Q, Chen X, Luo J. GSDS: a gene structure display server. Yi Chuan. 2007;29(8):1023-6.

56. Bai CK. Fatty acids analysis in Decaisnea insignis and Akebia trifoliate seed oil by GC-MS. Acta Bot Boreal-Occident Sin. 2007;27(5):1035-8.

\section{Publisher's Note}

Springer Nature remains neutral with regard to jurisdictional claims in published maps and institutional affiliations.

\section{Ready to submit your research? Choose BMC and benefit from}

- fast, convenient online submission

- thorough peer review by experienced researchers in your field

- rapid publication on acceptance

- support for research data, including large and complex data types

- gold Open Access which fosters wider collaboration and increased citations

- maximum visibility for your research: over $100 \mathrm{M}$ website views per year

At BMC, research is always in progress.

Learn more biomedcentral.com/submissions 